\title{
Abdominal Adiposity Quantification at MRI via Fuzzy Model-Based Anatomy Recognition \\ Yubing Tong ${ }^{1}$, J.K. Udupa ${ }^{1}$, D. Odhner ${ }^{1}$, Sanghun $\operatorname{Sin}^{2}$, R. Arens ${ }^{2}$ \\ ${ }^{1}$ Medical Image Processing Group, Dept. of Radiology, University of Pennsylvania, 423 Guardian \\ Drive, Blockley Hall, $4^{\text {th }}$ Floor, Philadelphia, PA 19104 \\ ${ }^{2}$ The Children's Hospital at Montefiore, Albert Einstein College of Medicine, Bronx, NY.
}

\begin{abstract}
In studying Obstructive Sleep Apnea Syndrome (OSAS) in obese children, the quantification of obesity through MRI has been shown to be useful. For large-scale studies, interactive or manual segmentation strategies become inadequate. Our goal is to automate this process to facilitate high throughput, precision, and accuracy and to eliminate subjectivity in quantification. In this paper, we demonstrate the adaptation, to this application, of a general body-wide Automatic Anatomy Recognition (AAR) system that is being developed separately. The AAR system has been developed based on existing clinical CT image data of 50-60 year-old male subjects and using fuzzy models of a large number of objects in each body region. The individual objects and their models are arranged in a hierarchy that is specific to each body region. In the application under consideration in this paper, we are primarily interested in only the skin boundary, and subcutaneous and visceral adipose region. Further, the image modality is MRI, and the study subjects are 8-17 year-old females. We demonstrate in this paper that, once such a full AAR system is built, it can be easily adapted to a new application by specifying the objects of interest, their hierarchy, and a few other application-specific parameters. Our tests based on MRI of 14 obese subjects indicate a recognition accuracy of about 2 voxels or better for both types of adipose regions. This seems quite adequate in terms of the initialization of model-based graph-cut (GC) and iterative relative fuzzy connectedness (IRFC) algorithms implemented in our AAR system for subsequent delineation of the objects. Both algorithms achieved low false positive volume fraction (FPVF) and high true positive volume fraction (TPVF), with IRFC performing better than GC.
\end{abstract}

Keywords: Segmentation, Object Recognition, Fuzzy models, Quantification of adiposity, Obstructive sleep apnea

\section{INTRODUCTION}

Mechanisms leading to Obstructive Sleep Apnea Syndrome (OSAS) in obese children are not well understood. In general, visceral obesity has been associated with metabolic syndrome and poor cardiovascular outcomes. It has been shown that visceral adiposity is more profound in obese sleep apneic adult subjects as compared to non-apneic obese subjects [1]. Previous research has also shown that Body Mass Index (BMI) alone does not differentiate between obese phenotypes but body composition (same BMI but different distribution of fat) in terms of the quantity of visceral and subcutaneous adipose tissue (VAT and SAT) components may suggest different phenotypes of obese subjects.

Manual or human assisted segmentation was previously used for the quantification of subcutaneous and visceral adiposity at MRI. For large-scale population studies, this becomes a hindrance. We used this application as a test-bed for studying the applicability of our general computerized automatic anatomy recognition (AAR) system. The goal of AAR is to identify and delineate the various organs in the body region automatically in a given patient image set for a specific body region. The approach of AAR should be as much as possible general and independent of the organs, body region, imaging modality, particular application, and the imaging protocols. Given the difficulties in image segmentation and the vagaries of clinical imaging scenarios, it is imperative that body-wide models representing to normal populations should be created to serve in AAR.

Our earlier work $[2,3]$ described methods for building such a general AAR system using fuzzy anatomy modeling principles to find natural and computationally efficient means of bringing anatomic knowledge about large assemblies of objects into image segmentation tasks. Particularly, we employed fuzzy concepts so as to bring prior knowledge into

Medical Imaging 2013: Biomedical Applications in Molecular, Structural, and Functional Imaging,

edited by John B. Weaver, Robert C. Molthen, Proc. of SPIE Vol. 8672, 86721R

(C) 2013 SPIE $\cdot$ CCC code: $1605-7422 / 13 / \$ 18 \cdot$ doi: 10.1117/12.2007938

Proc. of SPIE Vol. $867286721 \mathrm{R}-1$ 
graph-based segmentation approaches such as fuzzy connectedness [4]. The hierarchical arrangement inherent in the anatomic layout of organs were modeled and explored to make AAR effective and efficient. The general AAR approach consists of three main steps: (a) model generation from existing image data; (b) object recognition in a given image using the models; (c) delineation and quantification of the recognized objects. In this paper, we will demonstrate the adaptation of our AAR system for automation of the detection of the abdominal adipose regions at MRI of obese subjects based on fuzzy models of the AAR system previously created from a different image modality, namely CT, and a different patient group, namely 50-60 year old male subjects.

A brief description of the AAR method is given in Section 2, which includes fuzzy object modeling, recognition, as well as delineation methods. Recognition and delineation results are shown in Section 3 with quantitative and qualitative evaluation. Our conclusions are stated in Section 4.

\section{METHOD}

Our hypothesis was that if we had a previously created AAR system complete with body-wide anatomic models, then it should be possible to deploy the system for effective anatomy recognition in any application context involving a body region for which the system already includes models, no matter what the original image modality and acquisition protocol were. We have built a preliminary AAR system including several body regions based on existing routine clinical patient CT images [2,3]. It employs population-wise fuzzy anatomic models which codify the form, geographic layout, and inter-relationships among objects. Our AAR system includes fuzzy models of about 15 objects for the abdominal body region, which is our focus in this paper. The application in which we wish to test the previously built AAR system differs from the premise of the original AAR system in three ways: (i) a different image modality (MRI vs CT), (ii) different number of objects considered (just 2-3 vs the original 15), and (iii) different age group (8-17 female vs the original 50-60 male). Our system is preliminary and demonstrational and hence so far considered only the group of 50-60 male subjects. The proper way of handling (iii) is to have more subject groups at a sufficient "resolution" of the population variables in the future.

\subsection{Fuzzy object models}

In our AAR system, models are built and assembled by body region. For any body region $B$, its (fuzzy object) model $F O M(B)$ consists of five entities; $F O M(B)=(H, M, F M, \rho, \eta)$. $H$ here is a hierarchical order (tree) of the organs $O_{1}, \ldots$, $O_{\ell}$ in $B$ considered for inclusion in the model. $M=\left\{F M_{\ell}: 1 \leq \ell \leq L\right\}$ is a set of fuzzy models, one for each of the $L$ organs. $\rho$ represents parent-to-offspring relationship in $H . \lambda=\left\{\lambda_{\ell}: 1 \leq \ell \leq L\right\}, \lambda_{\ell}$ being scale factor range of organ $O_{\ell}$ over the population considered. $\eta$ is a host of measurements pertaining to organs in $B$ derived from the population.

In our application, $B=$ abdomen. The model $\operatorname{FOM}(B)$ we have built previously for the abdomen was derived from contrast enhanced CT images and consisted of $L=15$ objects, arranged in a certain hierarchy [3]. Since our focus in this paper is on SAT and VAT and since the body region boundary itself (which we will refer to as Skin) is important for defining SAT and VAT, we will consider just three objects $(L=3)$. The arrangement of the hierarchy $H$ in this case is as shown in Figure 1. The images we deal with in our application come from MRI, but we also tested on CT image data. The adaptations required were essentially in the form of constructing $H$ (which in this case is much simpler than the original) as well as making sure that the extent of the body region $B$ is defined in consistency with the definition used in the original AAR system. We will incorporate intelligent search strategies [5] in the future to carve out automatically the volume image corresponding to $B$ from a superset of slice volume data which contains the precise $B$ we have defined in the original AAR system. If the set of objects considered in the new application is a subset of the objects included in the original $\operatorname{FOM}(B)$ model, then the only change required is the specification of the new hierarchy, as described above. If, however, new objects are to be included, then the new hierarchy and the binary images representing to the new objects required for building their fuzzy models are also to be supplied. The system then automatically updates $F O M(B)$. 


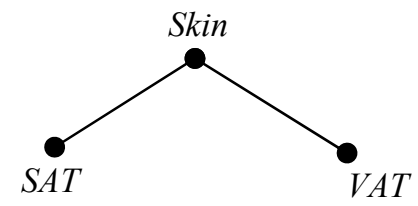

Figure 1. Hierarchy $H$ considered for $B$ in our application.

\subsection{Recognition in AAR}

For defining objects in a given image $I$, our AAR system takes two separate steps - recognition and delineation. Recognition (or global recognition) determines the optimal pose for the model (corresponding to each object) at which it is to be placed in $I$ so that the model falls as close as possible to the object in $I$. The delineation step uses the model information and the image information to optimally delineate the spatial extent of the object. For the final object definition in $I$ to be optimal, it is essential that the global recognition step becomes effective. There are several algorithms implemented for recognition in our system. They fall under two categories - those that do not require search, those that employ a search strategy to maximize recognition score or minimize energy. We call the former one-shot methods. For this application we have tested one method from the former and two methods from the latter category. The one-shot method implemented here estimates the optimum pose by first recognizing the root object (Skin) and delineating it and then placing other objects relative to the root object by using the inter-object relationship information $\rho$ available in the model. One of the search methods implemented optimizes a b-scale-based energy functional to find the best pose. Another searching method, optimum-threshold method, uses an optimum threshold interval determined for each object to minimize deviation with respect to the model. For MRI images, image intensity standardization is done before modeling to make sure that the intensity value for every subject will be roughly in the same range [6]. For test images also, intensity standardization has to be applied before the recognition step.

One-shot method: The pose of the model in $I$ is determined from the pose of the root object (skin boundary) and $\rho_{l, k}$. The root object is recognized and delineated by thresholding and searching for optimum fit with the model.

B-scale method: The energy function associated with $F M_{l}$ at pose $p$ in $I$ is $E(p)=\sum_{c \in Q_{p}} f_{b}(c)$, where $f_{b}(\mathrm{c})$ is the bscale value at voxel $c$, and $Q_{p}$ is the set of voxels, corresponding to pose $p$ of $F M_{l}$, at which the model membership is about 0.5 .

Optimum-threshold method: Given a specific organ $O_{k}$, we can fix the corresponding threshold interval $T H_{k}$ (optimum threshold) for it by checking all the training images. Then with that threshold, we segment the test image $I$ roughly which will include a good portion of the target organ although there may be some other neighboring organs or tissues but the organs outside this intensity threshold interval will not disturb our recognition process. The optimal pose is the one for which we get the minimum difference between modified model and the thresholded result.

\subsection{Delineation}

A delineation method is used to precisely describe the target organ after we have found where the target organ is in the test image by recognition. We have explored the fuzzy connectedness family of algorithms including the basic fuzzy connectedness algorithm and iterative relative fuzzy connectedness (IRFC) as well as graph cut in our previous research $[4,5]$. We have also proved seed robustness for fuzzy connectedness compared to graph cut which means that for FC or IRFC, we need only a few effective seed samples. Seeds are selected automatically by thresholding the fuzzy object model and the image simultaneously. The IRFC algorithm is used subsequently with a modification to take into account in addition to the image homogeneity and object feature components of affinity also a model-based component. 


\section{RESULTS}

The CT data from which the models were built consisted of image data from 28 normal subjects (voxel size $0.98 \times 0.98$ x $5.00 \mathrm{~mm}^{3}$ ) and the MRI data came from 14 obese patients (voxel size $0.71 \times 0.71 \times 6.0 \mathrm{~mm}^{3}$ ). Fuzzy object models were generated from the CT data sets for the three objects in the hierarchy shown in Figure 1. Figure 2 shows some recognition results where the model is overlaid on the original images at the time of recognition to show where the AAR system found the objects.

Quantitative recognition results are summarized in Table 1. The mean and standard deviation of the location errors (with respect to ground truth binary images obtained by interactive segmentation of the 14 data sets) and scale factor errors are listed in the table. The scale factor error is shown as a ratio of the estimated to the actual scale factor of the objects. The ideal values for these two error measures would be 0 and 1 , respectively.
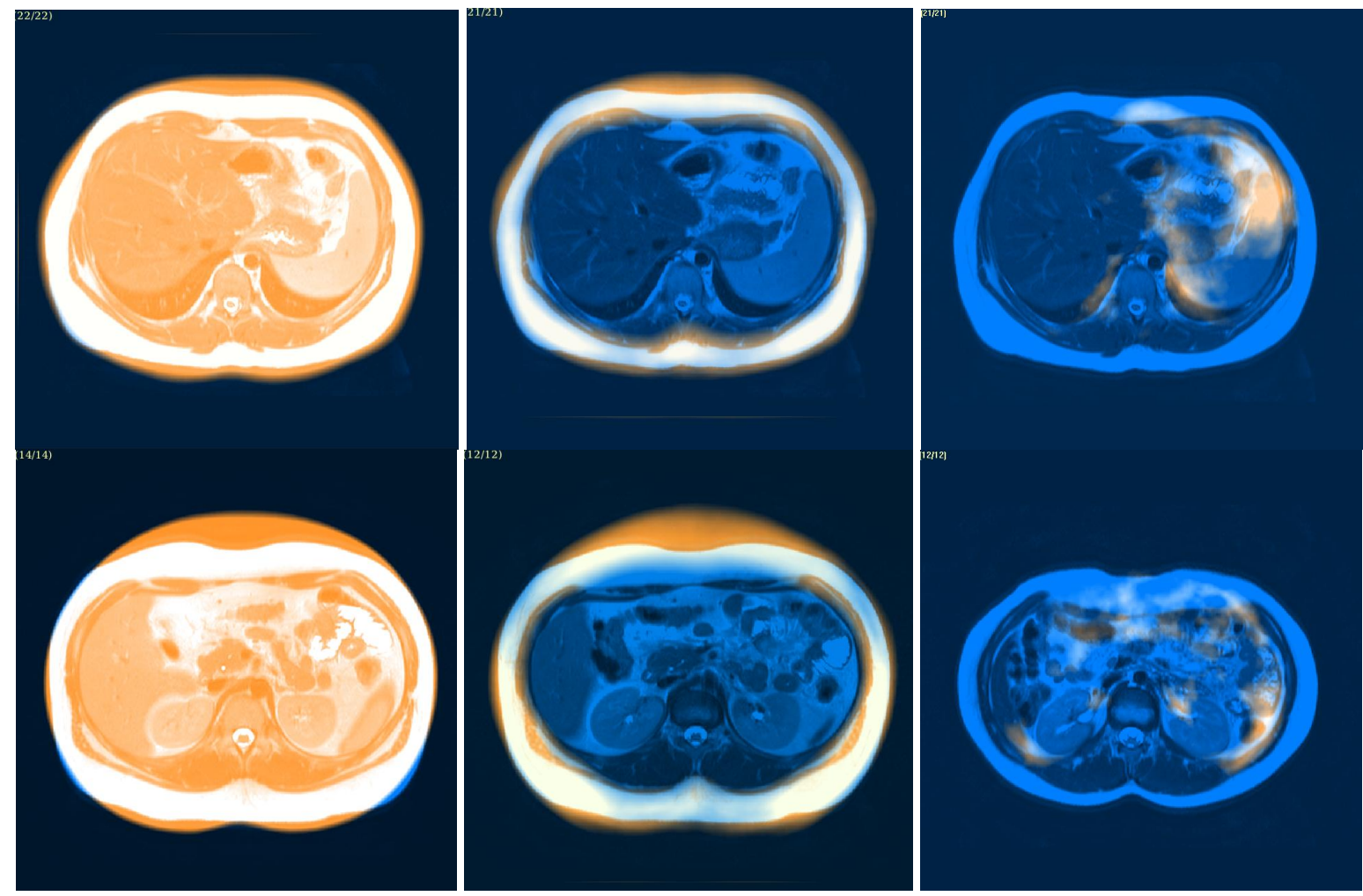

Figure 2. Some recognition results with the fuzzy object models overlaid on the original images. From left to right, top to bottom: Skin, SAT, and VAT derived from MRI data sets of 2 subjects.

Delineation results are shown in Figure 3 for Skin, SAT and VAT overlaid on the original gray images. In figure 4, the delineated result of SAT from IRFC is shown via volume rendering with 3DVIEWNIX and CAVASS software [7]. Delineation accuracies in terms of true positive (TP) and false positive (FP) volume fractions [8] are shown in Table 2. 
Table 1. Recognition results with average location error (Loc err. mean) and its standard deviation (Std) and the average scale factor error (Scale err. Mean) and its standard deviation (Std).

\begin{tabular}{|c|c|c|c|c|c|}
\hline & Error & Skin & SAT & VAT & Mean \\
\hline \multirow{4}{*}{ One-shot } & Loc err. mean & 4.08 & 14.11 & 17.88 & 12.02 \\
\cline { 2 - 6 } & Std & 2.59 & 5.01 & 8.58 & 5.39 \\
\cline { 2 - 6 } & Scale err. mean & 1.01 & 1.05 & 1.1 & 1.05 \\
\cline { 2 - 6 } & Std & 0.05 & 0.04 & 0.07 & 0.06 \\
\hline \multirow{4}{*}{ b-scale } & Loc err. mean & 3.51 & 14.51 & 17.62 & 11.88 \\
\cline { 2 - 6 } & Std & 2.33 & 4.65 & 7.98 & 4.99 \\
\cline { 2 - 6 } & Scale err. mean & 0.98 & 0.99 & 1.07 & 1.01 \\
\cline { 2 - 6 } & Std & 0.05 & 0.04 & 0.06 & 0.05 \\
\hline \multirow{4}{*}{$\begin{array}{c}\text { Optimum } \\
\text { threshold }\end{array}$} & Loc err. mean & 3.49 & 13.34 & 9.88 & 8.91 \\
\cline { 2 - 6 } & Scale err. mean & 1 & 1 & 0.95 & 0.98 \\
\cline { 2 - 6 } & Std & 0.01 & 0.02 & 0.03 & 0.02 \\
\hline
\end{tabular}

Table 2. Delineation results from GC and IRFC.

\begin{tabular}{|c|c|c||c|c|c|}
\hline \hline & & Skin & SAT & VAT & mean \\
\hline \hline \multirow{2}{*}{ GC } & TPVF & 0.92 & 0.85 & 0.83 & 0.87 \\
\cline { 2 - 6 } & FPVF & 0.03 & 0.02 & 0.05 & 0.03 \\
\hline \multirow{2}{*}{ IRFC } & TPVF & 0.95 & 0.93 & 0.90 & 0.93 \\
\cline { 2 - 6 } & FPVF & 0.00 & 0.01 & 0.03 & 0.02 \\
\hline
\end{tabular}



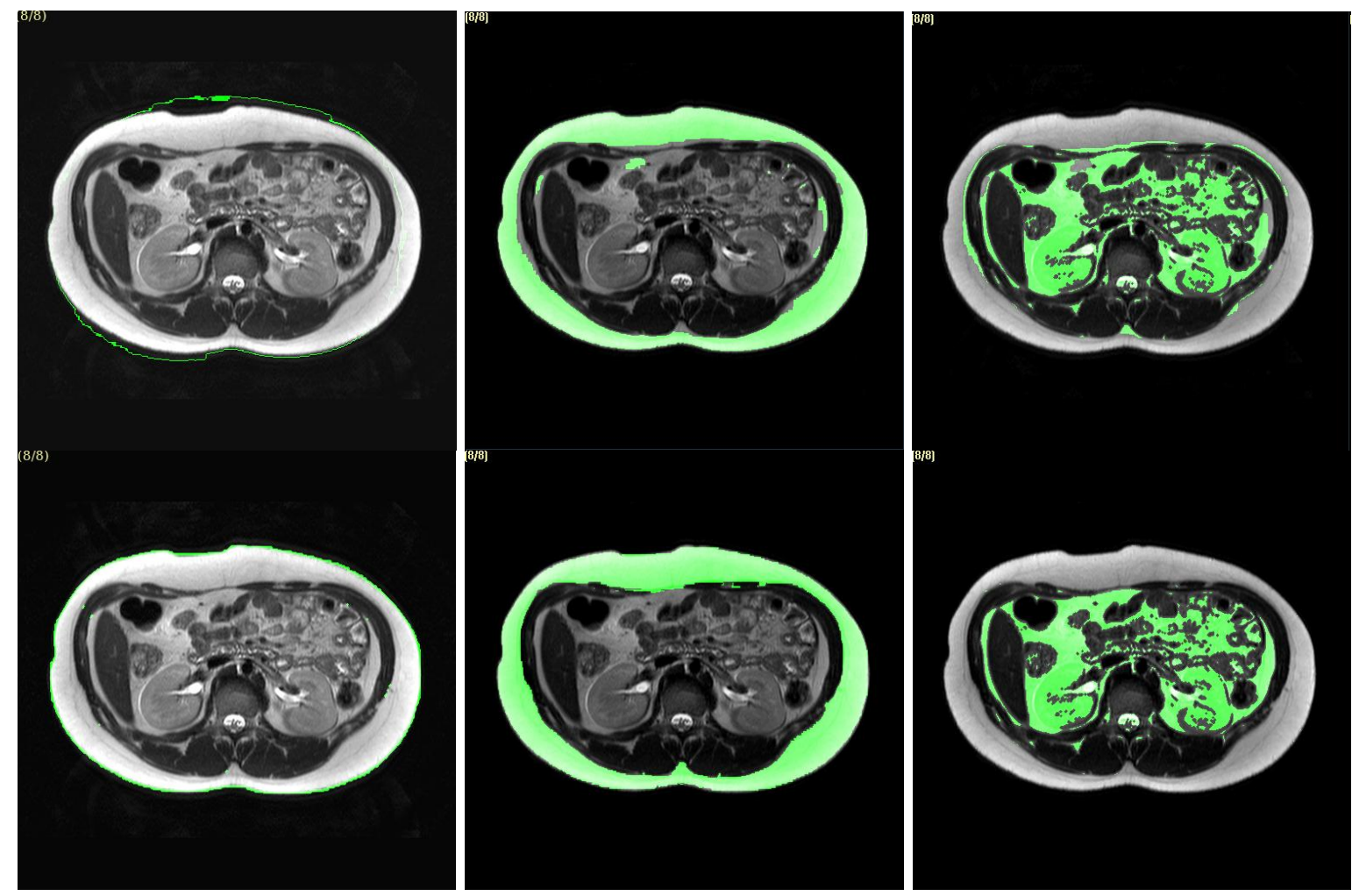

Figure 3. Delineation results for Skin, SAT, and VAT from GC (top row) and IRFC (bottom row). For Skin, only the outer boundary is shown.

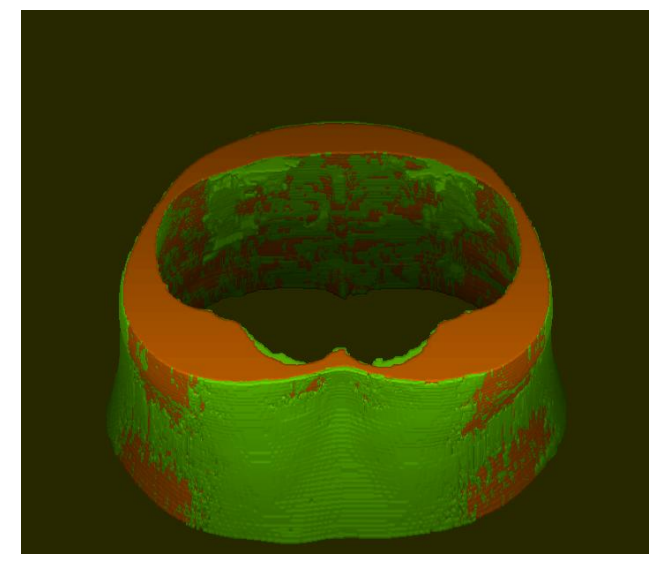

Figure 4. 3D visualization of subcutaneous adipose region delineated via IRFC and from manual segmentation. The red object is generated from ground truth data and the green from IRFC.

\section{CONCLUSION}

In the original FOM method, the body regions of focus were the thorax and abdomen based on contrast-enhanced CT images. We demonstrate in this paper that the same approach and software can be easily adapted to abdominal adiposity quantification and they work well in spite of the fact that prior knowledge came from a different population group and modality.

Our previous AAR system includes fuzzy models of about 15 objects for the abdominal body region but here the AAR method deals with only 3 objects with a very simple hierarchical structure and still it works effectively. The recognition 
results indicate that the average location error is about 2 voxels, which seems adequate to get high delineation accuracy. Delineation experiments show that model-based IRFC can achieve high true positive value and very low false positive value. The IRFC delineation method works better than the GC method.

In the future, we will study other hierarchical structures with additional objects to test if different hierarchical structures can improve the recognition and/or delineation results. Note that although our focus is only SAT and VAT as objects, other objects may provide better context for recognition [9]. In such a case, we do not really need to fully delineate these other objects.

\section{Acknowledgements}

The research reported here is supported by a DHHS grant HL105212.

\section{REFERENCES}

[1] Vgontzas AN., " Does obesity play a major role in the pathogenesis of sleep apnoea and its associated manifestations via inflammation, visceral adiposity and insulin resistance?" Arch Physiol Biochem 114, 211-223 (2008).

[2] Udupa, J.K., Odhner, D., Falcao, A.X., Ciesielski, K.C., Miranda, P.A.V., Matsumoto M., Grevea, G.J., Saboury, B., and Torigian, D.A., "Automatic Anatomy Recognition via Fuzzy Object Models," Proc. SPIE 8316, 5-8 (2012).

[3] Udupa, J.K., Odhner, D., Falcao, A.X., Ciesielski, K.C., Miranda, P.A.V., Matsumoto M., Grevea, G.J., Saboury, B., and Torigian, D.A., "Fuzzy object modeling," Proc. SPIE 7964, 1-10 (2011).

[4] Ciesielski, K.C,. and Udupa, J.K., Falcao, A.X., Miranda, P.A.V., " Comparison of fuzzy connectedness and graph cut segmentation algorithms," Proc. SPIE 7962, 3-10 (2011).

[5] Chen, X., Udupa, J.K., Alavi, A., and Torigian, D.A., "Automatic anatomy recognition via multiple object oriented active shape models, " Medical Physics 37, 6391-6401 (2010).

[6] Saha, P. and Udupa, J.K., " Scale-based fuzzy connected image segmentation: Theory, algorithm and validation," Computer Vision and Image Understanding, 77(2):145-174 (2000).

[7] Grevera, G., Udupa, J.K., Odhner, D., "CAVASS: a framework for medical imaging applications," Proc. SPIE 7497, MIPPR (2009).

[8] Udupa, J.K., LeBlancb, V.R., Imielinskab, C., Currief, L. M., Hirschg, B. E., Woodburnh, J., "A framework for evaluating image segmentation algorithms," Computerized Medical Imaging and Graphics, 30, 75-87 (2006).

[9] Matsumoto, M.M.S., Udupa, J.K., "Optimal hierarchy for fuzzy object models," Proc. SPIE, 8671 (2013). 